\title{
Nontrivial Critical Fixed Point for Replica-Symmetry-Breaking Transitions
}

\author{
Patrick Charbonneau ${ }^{1,2}$ and Sho Yaida, ${ }^{1, *}$ \\ ${ }^{1}$ Department of Chemistry, Duke University, Durham, North Carolina 27708, USA \\ ${ }^{2}$ Department of Physics, Duke University, Durham, North Carolina 27708, USA
}

(Received 18 July 2016; revised manuscript received 6 November 2016; published 26 May 2017)

\begin{abstract}
The transformation of the free-energy landscape from smooth to hierarchical is one of the richest features of mean-field disordered systems. A well-studied example is the de Almeida-Thouless transition for spin glasses in a magnetic field, and a similar phenomenon-the Gardner transition-has recently been predicted for structural glasses. The existence of these replica-symmetry-breaking phase transitions has, however, long been questioned below their upper critical dimension, $d_{u}=6$. Here, we obtain evidence for the existence of these transitions in $d<d_{u}$ using a two-loop calculation. Because the critical fixed point is found in the strong-coupling regime, we corroborate the result by resumming the perturbative series with inputs from a three-loop calculation and an analysis of its large-order behavior. Our study offers a resolution of the long-lasting controversy surrounding phase transitions in finite-dimensional disordered systems.
\end{abstract}

DOI: 10.1103/PhysRevLett.118.215701

Introduction.-Spontaneous symmetry breaking can dramatically change material properties. Breaking translational symmetry turns liquids into crystalline solids, breaking gauged phase symmetry gives rise to superconductivity, and breaking non-Abelian gauged symmetry endows elementary particles with mass. In a host of disordered models, a symmetry of the most peculiar type can break. Upon cooling, the mean-field free-energy of these systems develops a finite complexity; the number of metastable states grows exponentially with system size. The similitude between copies (replicas) of the system then depends on whether or not they belong to a same cluster of metastable states. In particular, right at the transition point, each replica of the system is on the brink of falling into one cluster or another, resulting in critical fluctuations of the similarity between uncoupled copies. Remarkably, such replica symmetry breaking (RSB) accounts for the emergence of glassiness in mean-field models ranging from liquids to optimization problems and neural networks [1]. Mean-field criticality, however, bears the seed of its own destruction. Below the upper critical dimension, $d_{u}$, violent critical fluctuations challenge the very validity of the approximation within which they were conceived. The existence of a continuous transition into an RSB phase in dimensions $d<$ $d_{u}$ is thus not a foregone conclusion, and its fate in disordered systems remains hotly debated [2].

An illustrious example of this dispute centers around mean-field models of spins with quenched impurities in an external magnetic field, known to exhibit a de AlmeidaThouless (dAT) transition [3]. This transition accompanies the emergence of continuous RSB with a hierarchically rough landscape, which eventually becomes fractal in the low-temperature limit [4]. Its upper critical dimension, $d_{u}=6$ [5], however, is well above 3 and the existence of the transition in real physical systems has long been questioned [6-13]. Recent advances in the mean-field description of structural glasses have unveiled a new facet of this problem. Solid glasses are also predicted to undergo a critical RSB transition - known as a Gardner transitionupon cooling, compressing, or shearing [4,14,15]. A growing body of evidence further relates the Gardner transition to the anomalous behavior of amorphous solids compared to their crystalline counterpart, and to a nontrivial critical scaling upon approaching the jamming limit [4,16-20]. The question of whether dAT and Gardner transitions survive finite-dimensional fluctuations has thus gained renewed impetus.

The impact of fluctuations on RSB transitions was first examined using the perturbative renormalization group (RG) approach that proved so successful for Ising and other universality classes. A loop expansion of the field theory appropriate for the dAT and Gardner universality class, however, finds that the critical fixed point is absent to lowest, one-loop order for $d<d_{u}$ [21-23]. This has led many to conclude that such transitions then either become discontinuous or simply vanish. Yet the lack of dimensional robustness is challenged by numerical evidence supporting the existence of a critical dAT transition in $d=4[24,25]$ and of a critical Gardner transition in $d=3$ [26,27]. An alternate interpretation is that the critical fixed point resides in the strong-coupling regime of the field theory for all $d$, thus preventing a one-loop calculation from identifying it. A precedent is the Caswell-Banks-Zaks (CBZ) fixed point of the non-Abelian gauge theory for elementary particles in $3+1$ space-time dimensions [29]. This fixed point is missed at one-loop order but captured at two-loop order. Although the CBZ fixed point generically lies in the strongcoupling regime, which falls beyond the designed range of 


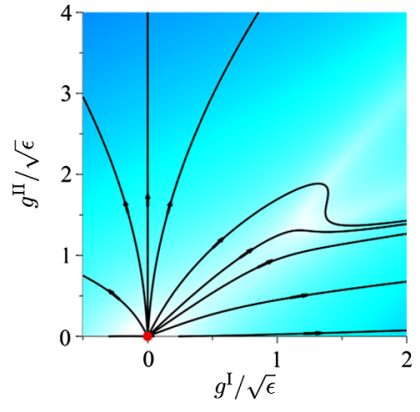

(a) One-loop RG, $d<6$

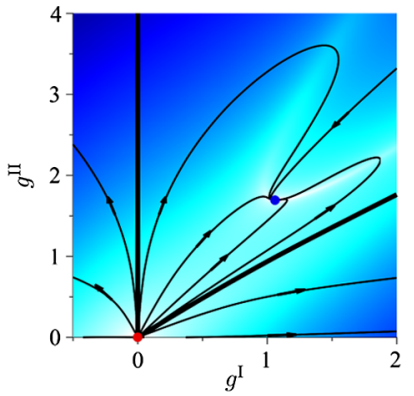

(b) Two-loop RG, $d=3$

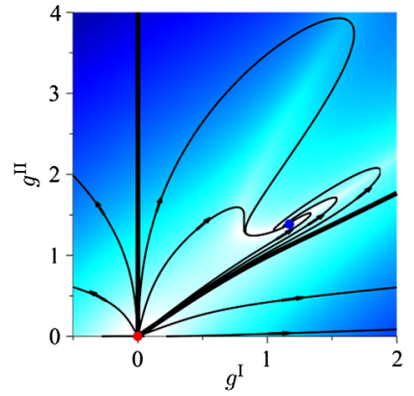

(c) Two-loop RG, $d=5$

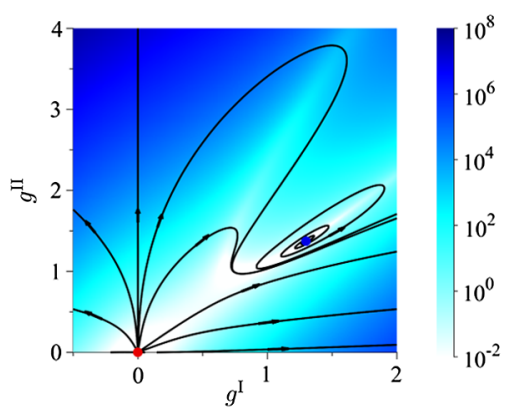

(d) Two-loop RG, $d=5.5$

FIG. 1. RG flows in the space of couplings for (a) the one-loop calculation in $d<6$ and the two-loop calculation in (b) $d=3$, (c) $d=5$, and (d) $d=5.5$. Arrows denote flow toward longer length scales; background shading denotes the intensity of the flow quantified by $\left(\beta^{\mathrm{I}}\right)^{2}+\left(\beta^{\mathrm{II}}\right)^{2}$, and normalized by $\epsilon^{-3}$ in (a). The Gaussian fixed point (red dot) is unstable for $d<6$. In (b) and (c) a nontrivial fixed point (blue dot) is stable and lies at strong couplings. Its basin of attraction is delineated by two thick lines: one precisely along $g^{\mathrm{I}}=0$ and the other approximately along $g^{\mathrm{I}} \approx g^{\mathrm{II}}$. Outside this basin, the flow runs toward infinity, which is often characteristic of discontinuous transitions. Note that for $d=5$, the flow spirals into the nontrivial fixed point, while for $d=5.5$ both fixed points are unstable.

a perturbative calculation, its existence has been corroborated by adiabatically connecting it to a perturbative fixed point [30], supported by lattice simulations even in the strong-coupling regime [31], and established beyond reasonable doubt in supersymmetric theories [32,33]. Twoloop calculations may thus find fixed points that are missed by one-loop analysis, but additional lines of evidence are then needed to confirm the result.

In this Letter, we present field-theoretic calculations that capture the physics of both dAT transitions in spin glasses and Gardner transitions in structural glasses. Like for the CBZ fixed point, our two-loop calculation identifies a critical fixed point for $d<d_{u}$ that is missed by the one-loop $\mathrm{RG}$ flow. Resummation of the perturbative series at threeloop order supplemented by an analysis of its large-order behavior further supports the robustness of this critical fixed point for the dAT-Gardner universality class.

Field-theory setup.-The finite-dimensional generalization of the mean-field Edwards-Anderson order parameter for glasses is the replicated overlap field, $q_{a b}(\mathbf{x})$. This field characterizes the similarity at positions $\mathbf{x}$ between pairs of distinct replicated configurations through an $n$-by- $n$ symmetric matrix with a null diagonal; the zero replica limit, $n \rightarrow 0$, is taken at the end of the calculations in order to properly average over disorder [34]. In general, field fluctuations can be subdivided into longitudinal, anomalous, and replicon modes [35,36]. At dAT and Gardner transitions only replicon modes become critical (massless); the other two remain short-ranged (massive) and can thus be neglected at long distances. We henceforth only focus on the replicon field, $\phi_{a b}(\mathbf{x})$, defined by the condition $\sum_{b=1}^{n} \phi_{a b}=$ 0 for all $a=1, \ldots, n$, thus leaving $n(n-3) / 2$ degrees of freedom.

In order to investigate the putative critical point, we seek infrared-stable fixed points of the RG flow within the critical surface on which the replicon field remains massless. Within this surface, the field theory is governed by the bare action, $S=\int d \mathbf{x} \mathcal{L}$, with [37]

$$
\begin{aligned}
\mathcal{L}= & \frac{1}{2} \sum_{a, b=1}^{n}\left(\nabla \phi_{a b}\right)^{2} \\
& -\frac{1}{3 !}\left(g_{\text {bare }}^{\mathrm{I}} \sum_{a, b=1}^{n} \phi_{a b}^{3}+g_{\text {bare }}^{\mathrm{II}} \sum_{a, b, c=1}^{n} \phi_{a b} \phi_{b c} \phi_{c a}\right),
\end{aligned}
$$

which is the most generic cubic action for replicon modes [22]. The effective description of the system then depends on the energy scale, $\mu$, probed. This dependence is encoded in the RG flow of dimensionless couplings, $g^{\mathcal{X}}(\mu)$ with $\mathcal{X} \in\{\mathrm{I}, \mathrm{II}\}$, that are related to bare couplings, $g_{\text {bare }}^{\mathcal{X}}$, in Eq. (1) [34]. The flow is governed by $\beta^{\mathcal{X}} \equiv \mu \partial g^{\mathcal{X}} / \partial \mu$, and stops at fixed points, where $\beta^{\mathrm{I}}\left(g_{\star}^{\mathrm{I}}, g_{\star}^{\mathrm{II}}\right)=\beta^{\mathrm{II}}\left(g_{\star}^{\mathrm{I}}, g_{\star}^{\mathrm{II}}\right)=0$. Note that for all $d$ a Gaussian fixed point with $g_{\star}^{\mathrm{I}}=g_{\star}^{\mathrm{II}}=0$ exists, but it is stable only for $d>d_{u}$ [21].

Two-loop RG.-Inspired by the CBZ fixed point, we compute the $\beta$ functions to two-loop order for the replica field theory in Eq. (1), using the dimensional regularization scheme [29,34,38-43]. As expected [21-23], no stable fixed point can be found at one-loop order for $d<6$ [Fig. 1(a)]. For $d<d_{0} \approx 5.41$, however, the two-loop RG flow locates a stable fixed point with a finite basin of attraction [Figs. 1(b) and 1(c)]. A system lying within this basin eventually approaches the fixed point upon rescaling and is thus critical. By contrast, a system that remains outside the basin cannot continuously transition into an RSB phase, and may instead exhibit a discontinuous transition. Remarkably, the boundary of the basin is closely approximated by the tree-level condition for a critical transition into a RSB phase, i.e., $1<g^{\mathrm{II}} / g^{\mathrm{I}}<\infty$ [44].

The eigenvalues, $\lambda_{1}$ and $\lambda_{2}$, of

$$
\left.\left[\begin{array}{ll}
\frac{\partial \beta^{\mathrm{I}}}{\partial g^{\mathrm{I}}} & \frac{\partial \beta^{\mathrm{I}}}{\partial g^{\mathrm{II}}} \\
\frac{\partial \beta^{\mathrm{II}}}{\partial g^{\mathrm{I}}} & \frac{\partial \beta^{\mathrm{II}}}{\partial g^{\mathrm{II}}}
\end{array}\right]\right|_{\left(g^{\mathrm{I}}, g^{\mathrm{II}}\right)=\left(g_{\star}^{\mathrm{I}}, g_{\star}^{\mathrm{II}}\right)}
$$




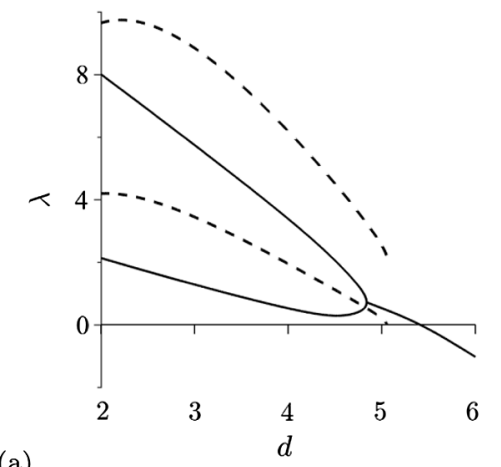

(a)

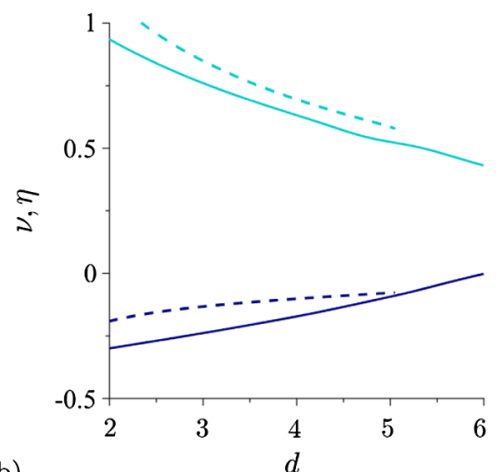

(b)

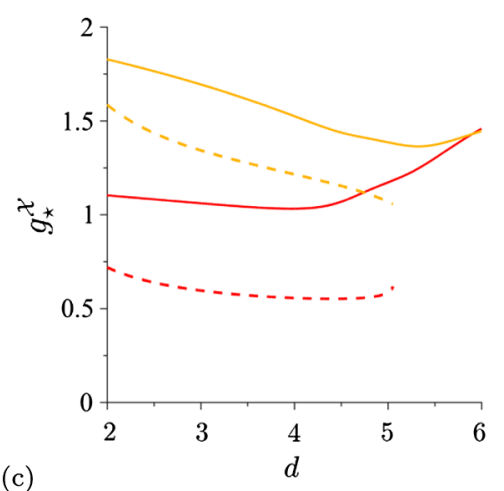

FIG. 2. Critical parameters at the nontrivial fixed point derived within two-loop (solid lines) and three-loop with Borel resummation (dashed lines) RG schemes as functions of the spatial dimension $d$. (a) Real parts of the stability exponents around the fixed point within the critical surface, $\lambda_{1}$ and $\lambda_{2}$. (b) Critical exponents, $\nu$ (cyan) and $\eta$ (navy blue). (c) Fixed-point values of running couplings, $g^{\mathrm{I}}$ (red) and $g^{\mathrm{II}}$ (orange). At two-loop order, the nature of the fixed point changes at $d_{s} \approx 4.84$ and $d_{0} \approx 5.41$. The two stability exponents merge at $d=d_{s}$, at which point they acquire imaginary parts; hence, the flow spirals into the (stable) fixed point [Fig. 1(c)], while for $d>d_{0}$ the real part of these eigenvalues becomes negative and the flow spirals out of the (unstable) fixed point [Fig. 1(d)]. Upon inclusion of threeloop contributions, Borel resummation indicates that the fixed point is robustly stable for $d \lesssim 5.05$ but does not exhibit any spiraling flow.

give the stability exponents that control subleading corrections from irrelevant deformations near the critical point. Figure 2(a) indicates that these exponents acquire an imaginary component for $d>d_{s} \approx 4.84$; hence, the RG flow then spirals toward the fixed point [Fig. 1(c)]. As has been observed in other disordered systems [45-47], such complex exponents can emerge from the nonunitarity of the replica field theory, and give rise to an oscillatory decay of the appropriate correlation functions in the critical region. Conformality gets lost with the change in the spiral direction at $d=d_{0}$ and no stable fixed point can be found for $d \in\left(d_{0}, d_{u}\right)$ [Fig. 1(d)]. In the absence of additional nontrivial fixed points with which to collide [48], this scenario provides a natural mechanism for exchanging dominance between the Gaussian and the genuinely nonperturbative fixed points as one goes from $d>d_{u}$ down to physical dimensions.

We also compute the critical exponents, $\nu$ and $\eta$, that govern the divergence of the correlation length and the decay of two-point correlation functions at the critical point, respectively [Fig. 2(b)]. The former is obtained from the relevant deformation by the quadratic coupling that drives the system away from the critical surface. Estimates of $\nu$ and $\eta$ agree qualitatively with the trend observed in $d=4$ simulations [24]; $\eta$ is negative and $\nu$ is larger than its mean-field value, $\nu_{\mathrm{MF}}=\frac{1}{2}$.

Resummation.-Because the critical couplings are of order unity for all $d<d_{u}$ [Fig. 2(c)], resummation is needed to assess the existence of the fixed point. (Without a careful resummation, even the $d \leq 3$ WilsonFisher fixed point for the Ising universality class disappears [49].) A field-theoretic perturbative series is indeed generically not convergent but rather asymptotic. More precisely, a formal series in terms of the coupling constant,

$$
f\left(g^{2}\right)=\sum_{k} f_{k} g^{2 k}
$$

typically has coefficients that exhibit a factorial growth, i.e., $f_{k} \sim k !(-1 / A)^{k}$, with a large-order constant $A$ given in terms of the saddle-point action [50,51]. Although a truncation to the first couple of terms may yield a good approximation in the weak-coupling regime, the series itself is not mathematically well defined.

Borel resummation is the most common scheme used to give epistemological traction to a fixed point. The approach starts from the observation that a Borel transform, $\tilde{f}_{B}\left(g^{2}\right) \equiv \sum_{k}\left(f_{k} / k !\right) g^{2 k}$, has a finite radius of convergence, $|A|$. Using the identity $k !=\int_{0}^{\infty} d t e^{-t} t^{k}$ the original series [Eq. (3)] can formally be expressed as $f\left(g^{2}\right)=$ $\int_{0}^{\infty} d t e^{-t} \tilde{f}_{B}\left(t g^{2}\right)$. The analytic continuation of the Borel transform onto the whole positive axis then unambiguously defines the function $f$. There is typically no problem to this analytic continuation when $A>0$; hence, the series is then deemed Borel summable.

In order to adapt the above scheme to a replica field theory with two cubic couplings, we define $\left(g^{\mathrm{I}}, g^{\mathrm{II}}\right) \equiv$ $g(\cos \theta, \sin \theta)$ and regroup the double series, with the power of $g^{2}$ counting loop order:

$$
\begin{aligned}
f\left(g^{\mathrm{I}}, g^{\mathrm{II}}\right) & =\sum_{k_{1}, k_{2}=0 ; k_{1}+k_{2}=\text { even }}^{\infty} f_{k_{1}, k_{2}}\left(g^{\mathrm{I}}\right)^{k_{1}}\left(g^{\mathrm{II}}\right)^{k_{2}} \\
& =\sum_{k=0}^{\infty} g^{2 k}\left[\sum_{k_{1}=0}^{2 k} f_{k_{1}, 2 k-k_{1}}(\cos \theta)^{k_{1}}(\sin \theta)^{2 k-k_{1}}\right] \\
& \equiv \sum_{k=0}^{\infty} f_{k}(\theta) g^{2 k} .
\end{aligned}
$$


The Borel-summability of the series is then governed by the angle-dependent large-order behavior $f_{k}(\theta) \sim$ $k ![-1 / A(\theta)]^{k}$. Consequently, as has been observed for the Abelian gauge theory with background fields [52], Borel-summability depends on the ratio of two couplings, as encoded in the saddle-point solution to the classical equations of motion for replicons [53].

Among nontrivial saddles, we assume $[55,56]$ that the saddle of the form

$$
\phi_{a b}^{\star}(\mathbf{x} ; \theta)=\frac{1}{g} F(\mathbf{x}) v_{a b}^{(\theta)}
$$

dictates the value $A(\theta)$. Here, $F$ is a spherically symmetric function that solves

$$
\nabla^{2} F=F-F^{2}
$$

obtained numerically through the pseudospectral method [57,58], and $v_{a b}^{(\theta)}$ is the replicon component of the Parisi RSB ansatz [1,59-61]. Computing the action of the resulting saddle [34] indicates that a solution exists if and only if $1<\tan \theta<\infty$, with

$$
A(\theta)=\frac{c_{d}}{\cos ^{2} \theta(\tan \theta-1)},
$$

where $c_{d}$ is a $d$-dependent positive constant. The series is thus Borel summable within the wedge $1<g^{\mathrm{II}} / g^{\mathrm{I}}<\infty$, consistent with the mean-field consideration [44] and the two-loop basin of attraction obtained in Fig. 1. This result thus validates our perturbative treatment of the strongcoupling regime within the basin of attraction.

Given the large-order behavior at hand, we further compute the critical properties of the fixed point by resumming the three-loop series, analytically continuing the Borel transform through the conformal mapping $[34,62]$. Comparing the two-loop and the resummation results upon inclusion of three-loop contributions [34] (Fig. 2) confirms that the fixed point is robustly conserved for $d \lesssim 5.05$. The critical exponents from the two schemes further qualitatively agree with one another.

Conclusion.-The nontrivial critical fixed point identified here governs both dAT and Gardner transitions in $d<d_{u}$. An RSB transition for the underlying universality class is thus possible over a broader $d$ range than previously thought [67-69]. The RG flow diagrams (Fig. 1) and the large-order behavior, however, make it clear that not all microscopic models belong to the basin of the attraction of the critical fixed point. This realization offers a possible explanation for the absence of dAT criticality in the Edwards-Anderson model in $d=3$. The model may simply remain outside the basin of attraction, and thus be governed either by a discontinuous transition into the RSB phase or by the two-state droplet picture [6-10]. Enlarging the range of disordered spin systems used for studying RSB criticality would clarify this last point.

Our results further highlight various future research directions. First, they guide efforts in systematizing nonperturbative RG methods [70] and controlling conformal bootstrap techniques for nonunitary theories [71-74]. Both approaches should find the nontrivial critical fixed point when applied to the replica field theory. Second, conflicting results have been obtained for the lower critical dimension, $d_{1}$, from a heuristic interface argument [75] and from a correlation-function argument [76]. The dimensional dependence of the infrared divergence associated with soft modes thus deserves further scrutiny. Third, extending the current approach will enable the study of the RG trajectory between the critical point identified here and the multicritical fixed point found perturbatively for the spin-glass transition in the absence of an external magnetic field [5], where longitudinal and anomalous modes become massless concurrently with the replicons.

Data relevant to this work can be accessed by following the link in Ref. [77].

We thank Giulio Biroli, Gerald V. Dunne, Atsushi Ikeda, Shamit Kachru, Jaehoon Lee, Michael A. Moore, Giorgio Parisi, Stephen H. Shenker, Mithat Ünsal, and Pierfrancesco Urbani for stimulating discussions and suggestions. This work was supported by a grant from the Simons Foundation (No. 454937, P. C.).

*sho.yaida@duke.edu

[1] M. Mézard, G. Parisi, and M. Virasoro, Spin Glass Theory and Beyond (World Scientific, Singapore, 1987).

[2] G. Parisi, Field theory and the physics of disordered systems, in Proceedings of Science, Hector Rubinstein Memorial Symposium: Quarks, Strings and the Cosmos, HRMS 2010, Stockholm, Sweden (2010).

[3] J. R. L. de Almeida and D. J. Thouless, Stability of the Sherrington-Kirkpatrick solution of a spin glass model, J. Phys. A 11, 983 (1978).

[4] P. Charbonneau, J. Kurchan, G. Parisi, P. Urbani, and F. Zamponi, Fractal free energy landscapes in structural glasses, Nat. Commun. 5, 3725 (2014).

[5] A. B. Harris, T. C. Lubensky, and J.-H. Chen, Critical Properties of Spin Glasses, Phys. Rev. Lett. 36, 415 (1976).

[6] W. L. McMillan, Scaling theory of Ising spin glasses, J. Phys. C 17, 3179 (1984).

[7] D. S. Fisher and D. A. Huse, Ordered Phase of Short-Range Ising Spin Glasses, Phys. Rev. Lett. 56, 1601 (1986).

[8] D. A. Huse and D.S. Fisher, Pure states in spin glasses, J. Phys. A 20, L997 (1987).

[9] D. S. Fisher and D. A. Huse, Equilibrium behavior of the spin-glass ordered phase, Phys. Rev. B 38, 386 (1988).

[10] A. J. Bray and M. A. Moore, Scaling theory of the ordered phase of spin glasses, in Heidelberg Colloquium on Glassy Dynamics: Proceedings of a Colloquium on Spin Glasses, Optimization and Neural Networks Held at the University of Heidelberg June 9-13, 1986, edited by J. L. van Hemmen 
and I. Morgenstern (Springer, Berlin, Heidelberg, 1987), p. 121.

[11] C. M. Newman and D. L. Stein, Nature of Ground State Incongruence in Two-Dimensional Spin Glasses, Phys. Rev. Lett. 84, 3966 (2000).

[12] C. M. Newman and D. L. Stein, Ordering and broken symmetry in short-ranged spin glasses, J. Phys. Condens. Matter 15, R1319 (2003).

[13] M. A. Moore and A. J. Bray, Disappearance of the de Almeida-Thouless line in six dimensions, Phys. Rev. B 83, 224408 (2011).

[14] C. Rainone, P. Urbani, H. Yoshino, and F. Zamponi, Following the Evolution of Hard Sphere glasses in Infinite Dimensions under External Perturbations: Compression and Shear Strain, Phys. Rev. Lett. 114, 015701 (2015).

[15] G. Biroli and P. Urbani, Breakdown of elasticity in amorphous solids, Nat. Phys. 12, 1130 (2016).

[16] E. DeGiuli, E. Lerner, C Brito, and M. Wyart, Force distribution affects vibrational properties in hard-sphere glasses, Proc. Natl. Acad. Sci. U.S.A. 111, 17054 (2014).

[17] E. DeGiuli, E. Lerner, and M. Wyart, Theory of the jamming transition at finite temperature, J. Chem. Phys. 142, 164503 (2015).

[18] P. Charbonneau, E. I. Corwin, G. Parisi, and F. Zamponi, Jamming Criticality Revealed by Removing Localized Buckling Excitations, Phys. Rev. Lett. 114, 125504 (2015).

[19] P. Charbonneau, E. I. Corwin, G. Parisi, A. Poncet, and F. Zamponi, Universal Non-Debye Scaling in the Density of States of Amorphous Solids, Phys. Rev. Lett. 117, 045503 (2016).

[20] A. Seguin and O. Dauchot, Experimental Evidence of the Gardner Phase in a Granular Glass, Phys. Rev. Lett. 117, 228001 (2016).

[21] A. J. Bray and S. A. Roberts, Renormalisation-group approach to the spin glass transition in finite magnetic fields, J. Phys. C 13, 5405 (1980).

[22] I. R. Pimentel, T. Temesvari, and C. De Dominicis, Spin glass transition in a magnetic field: A renormalization group study, Phys. Rev. B 65, 224420 (2002).

[23] P. Urbani and G. Biroli, Gardner transition in finite dimensions, Phys. Rev. B 91, 100202(R) (2015).

[24] R. A. Baños et al., Thermodynamic glass transition in a spin glass without time-reversal symmetry, Proc. Natl. Acad. Sci. U.S.A. 109, 6452 (2012).

[25] L. Leuzzi, G. Parisi, F. Ricci-Tersenghi, and J. J. RuizLorenzo, Ising Spin-Glass Transition in a Magnetic Field Outside the Limit of Validity of Mean-Field Theory, Phys. Rev. Lett. 103, 267201 (2009).

[26] L. Berthier, P. Charbonneau, Y. Jin, G. Parisi, B. Seoane, and F. Zamponi, Growing timescales and length scales characterizing vibrations of amorphous solids, Proc. Natl. Acad. Sci. U.S.A. 113, 8397 (2016).

[27] Strictly speaking, the Gardner transition exists in finite dimensions only in the high-overlap state of the $\varepsilon$-quenched Franz-Parisi potential for $\varepsilon>\varepsilon_{\mathrm{c}}\left(T, T^{\prime}\right)$ [28]. In the limit $\varepsilon \rightarrow 0^{+}$, in which typical simulations and experiments are carried out, the high-overlap glass states are only metastable. The time scale to escape from these metastable states can, however, be made extremely long, and vestiges of the Gardner transition can thus be well preserved.
[28] S. Franz and G. Parisi, Recipes for metastable states in spin glasses, J. Phys. I (France) 5, 1401 (1995).

[29] W. E. Caswell, Asymptotic Behavior of Non-Abelian Gauge Theories to Two-Loop Order, Phys. Rev. Lett. 33, 244 (1974).

[30] T. Banks and A. Zaks, On the phase structure of vector-like gauge theories with massless fermions, Nucl. Phys. B196, 189 (1982).

[31] L. D. Debbio, The conformal window on the lattice, Proc. Sci. LATTICE2010 (2010) 004.

[32] N. Seiberg, Electric-magnetic duality in supersymmetric non-Abelian gauge theories, Nucl. Phys. B435, 129 (1995).

[33] M. J. Strassler, The duality cascade, Progress in String Theory (World Scientific, Singapore, 2005), p. 419.

[34] See Supplemental Material at http://link.aps.org/ supplemental/10.1103/PhysRevLett.118.215701 for detailed discussions on the replica field theory formalism, the two-loop RG calculations of $\beta$ functions and critical exponents, and the three-loop RG calculations with resummations.

[35] A. J. Bray and M. A. Moore, Replica symmetry and massless modes in the Ising spin glass, J. Phys. C 12, 79 (1979).

[36] H. Nishimori, Statistical Physics of Spin Glasses and Information Processing: An Introduction (Oxford University Press, New York, 2001).

[37] Note that cubic couplings are sometimes also denoted as $g_{1} \equiv g^{\mathrm{II}}$ and $g_{2} \equiv g^{\mathrm{I}}$, with possible differences in overall factors of order 1 .

[38] G. 't Hooft and M. Veltman, Regularization and renormalization of gauge fields, Nucl. Phys. B44, 189 (1972).

[39] G. 't Hooft, Dimensional regularization and the renormalization group, Nucl. Phys. B61, 455 (1973).

[40] G. 't Hooft, An algorithm for the poles at dimension four in the dimensional regularization procedure, Nucl. Phys. B62, 444 (1973).

[41] D. J. Amit, Renormalization of the Potts model, J. Phys. A 9, 1441 (1976).

[42] O. F. d. A. Bonfirm, J. E. Kirkham, and A. J. McKane, Critical exponents for the percolation problem and the Yang-Lee edge singularity, J. Phys. A 14, 2391 (1981).

[43] J. A. Gracey, Four loop renormalization of $\phi^{3}$ theory in six dimensions, Phys. Rev. D 92, 025012 (2015).

[44] M. E. Ferrero, G. Parisi, and P. Ranieri, Fluctuations in a spin-glass model with one replica symmetry breaking, J. Phys. A 29, L569 (1996).

[45] A. Aharony, Critical properties of random and constrained dipolar magnets, Phys. Rev. B 12, 1049 (1975).

[46] J.-H. Chen and T. C. Lubensky, Mean field and $\varepsilon$-expansion study of spin glasses, Phys. Rev. B 16, 2106 (1977).

[47] A. Weinrib and B.I. Halperin, Critical phenomena in systems with long-range-correlated quenched disorder, Phys. Rev. B 27, 413 (1983).

[48] D. B. Kaplan, J.-W. Lee, D. T. Son, and M. A. Stephanov, Conformality lost, Phys. Rev. D 80, 125005 (2009).

[49] G. A. Jr. Baker, B. G. Nickel, M. S. Green, and D. I. Meiron, Ising-Model Critical Indices in Three Dimensions from the Callan-Symanzik Equation, Phys. Rev. Lett. 36, 1351 (1976). 
[50] L. N. Lipatov, Divergence of the perturbation-theory series and pseudoparticles, JETP Lett. 25, 104 (1977).

[51] E. Brézin, J. C. Le Guillou, and J. Zinn-Justin, Perturbation theory at large order. I. the $\varphi^{2 N}$ interaction, Phys. Rev. D 15, 1544 (1977).

[52] G. V. Dunne, Heisenberg-Euler effective Lagrangians: Basics and extensions, in From Fields to Strings: Circumnavigating Theoretical Physics, edited by M. Shifman, A. Vainshtein, and J. Weather (World Scientific, Singapore, 2005), Vol. 1.

[53] We can derive the equations of motion by introducing the Lagrange multiplier field [54], imposing the replicon constraints [34].

[54] S. Yaida, Instanton calculus of Lifshitz tails, Phys. Rev. B 93, 075120 (2016).

[55] S. R. Coleman, V. Glaser, and A. Martin, Action minima among solutions to a class of Euclidean scalar field equations, Commun. Math. Phys. 58, 211 (1978).

[56] J. Yeo, M. A. Moore, and T. Aspelmeier, Nature of perturbation theory in spin glasses, J. Phys. A 38, 4027 (2005).

[57] L. N. Trefethen, Spectral Methods in MATLAB (Oxford University Press, New York, 2000).

[58] A. Adams, T. Anous, J. Lee, and S. Yaida, Glassy slowdown and replica-symmetry-breaking instantons, Phys. Rev. E 91, 032148 (2015).

[59] G. Parisi, Infinite Number of Order Parameters for Spin Glasses, Phys. Rev. Lett. 43, 1754 (1979).

[60] T. Castellani and A. Cavagna, Spin-glass theory for pedestrians, J. Stat. Mech. (2005) P05012.

[61] F. Denef, TASI Lectures on Complex Structures, String Theory and Its Applications (World Scientific, Singapore, 2011), p. 407.

[62] See Refs. [63-66] for possible improvements on the resummation scheme.

[63] E. Brézin and G. Parisi, Critical exponents and large-order behavior of perturbation theory, J. Stat. Phys. 19, 269 (1978).
[64] J. C. Le Guillou and J. Zinn-Justin, Critical exponents from field theory, Phys. Rev. B 21, 3976 (1980).

[65] J. Zinn-Justin, Summation of divergent series: Orderdependent mapping, Applied Numerical Mathematics 60 , 1454 (2010).

[66] M. Ünsal and G. V. Dunne, What is QFT? Resurgent transseries, Lefschetz thimbles, and new exact saddles, Proc. Sci., LATTICE2015 (2015) 010.

[67] G. Parisi and T. Temesvári, Replica symmetry breaking in and around six dimensions, Nucl. Phys. B858, 293 (2012).

[68] M. C. Angelini and G. Biroli, Spin Glass in a Field: a New Zero-Temperature Fixed Point in Finite Dimensions, Phys. Rev. Lett. 114, 095701 (2015).

[69] M. C. Angelini and G. Biroli, Real space renormalization group theory of disordered models of glasses, Proc. Natl. Acad. Sci. U.S.A. 114, 3328 (2017).

[70] C. Wetterich, Exact evolution equation for the effective potential, Phys. Lett. B 301, 90 (1993).

[71] R. Rattazzi, V. S. Rychkov, E. Tonni, and A. Vichi, Bounding scalar operator dimensions in 4D CFT, J. High Energy Phys. 12 (2008) 031.

[72] S. El-Showk, M. F. Paulos, D. Poland, S. Rychkov, D. Simmons-Duffin, and A. Vichi, Solving the 3d ising model with the conformal bootstrap, Phys. Rev. D 86, 025022 (2012).

[73] F. Gliozzi, Constraints on Conformal Field Theories in Diverse Dimensions from the Bootstrap Mechanism, Phys. Rev. Lett. 111, 161602 (2013).

[74] S. El-Showk, M. F. Paulos, D. Poland, S. Rychkov, D. Simmons-Duffin, and A. Vichi, Solving the 3d ising model with the conformal bootstrap II. c-minimization and precise critical exponents, J. Stat. Phys. 157, 869 (2014).

[75] S. Franz, G. Parisi, and M. A. Virasoro, Interfaces and lower critical dimension in a spin glass model, J. Phys. I (France) 4, 1657 (1994).

[76] C. De Dominicis and I. Kondor, On spin glass fluctuations, J. Phys. Lett. 45, 205 (1984).

[77] DOI: 10.7924/G86Q1V5C. 